\title{
A Collaborative Filtering Algorithm Combined with User Habits for Rating
}

\author{
$\operatorname{Min} \mathrm{Li}^{1}$ \\ ${ }^{1}$ Computer Center \\ East China Normal University \\ Shanghai 200062, China \\ 51131211009@ecnu.cn
}

\author{
Kai Zheng ${ }^{2 *}$ \\ ${ }^{2}$ Computer Center \\ East China Normal University \\ Shanghai 200062, China \\ * Corresponding Author
}

\begin{abstract}
Collaborative filtering is one of the most successful and widely used technologies in personalized recommendation systems. This paper proposed a novel algorithm combined with user habits for rating as the conventional method leads lower accuracy relatively. In order to reveal the hidden relationship between users, the new algorithm not only reserves the traditional measure but also takes Bhattacharyya Coefficient and entropy into account while calculating the user similarities. Experiment results show the new algorithm outperforms the conventional method.
\end{abstract}

Keywords-Collaborative Filtering; Personalized Recommendation; User Habits for Rating; Bhattacharyya Coefficient; Entropy

\section{INTRODUCTION}

The advancement of the Internet helps promote the arrival of the information age, which has brought users a lot of conveniences during daily life. Meanwhile, the cumulative data is growing in exponential form so that it leads to "information overload" problem which results in that users can hardly find what they really need in limited time and energy. In order to tackle this issue, recommender systems come into being.

The study of recommender systems begins in the mid$1990 s^{[1]}$. Collaborative filtering (CF) is one of the most successful and widely used technologies in personalized recommend systems. Sarwar et al. ${ }^{[2]}$ proposed an item based CF as the similarity between items is more reliable than the similarity between users. Koren et al. ${ }^{[3]}$ applied singular value decomposition to the recommender systems in order to reduce the dimensionality of sparse ratings matrices. A PIP measure ${ }^{[4]}$ was put forward by Hyung which considered three aspects to user ratings: proximity, impact and popularity. Amatriain ${ }^{[5]}$ et al. proposed a new algorithm based on experts which had a better performance as the algorithm only calculated the similarity between user and experts.

Traditional measures including Cosine coefficient and Pearson correlation coefficient don't show satisfactory performance as these methods just only take the items both rated by two users into account. Few common rated ite ms between two users always cause the deviation when user similarity is calculated. This paper proposed a new algorithm which considers conventional measures and user habits for rating simultaneously. User habits similarity is measured by two factors: Bhattacharyya coefficient and entropy. Bhattacharyya coefficient indicates the degree of overlapping between user ratings while entropy indicates the dispersion degree of ratings voted by users. Experiments are implemented on the real data set MovieLens. In comparis on with the traditional measures, the novel algorithm show better recommended performance.

\section{CONVENTIONAL COLLABORATIVE FILTERING ALGORITHM BASED ON USERS}

\section{A. Build user-item rating matrix}

According to the user ratings for the items, build a user-item rating matrix $R(m, n)$ as Table I. shows below. $m$ represents the number of users, $n$ represents the number of items, $r_{i, j}$ denotes the rating of item $j$ by user $i$. In MovieLens dataset, users can assign item numeric ratings in the range $1-5$.

TABLE I. USER-ITEM RATING MATRIX

\begin{tabular}{|c|c|c|c|c|c|}
\hline & Item 1 & $\cdots \cdots$ & Item $\mathrm{j}$ & $\cdots \cdots$ & Item $\mathrm{n}$ \\
\hline User 1 & $\mathrm{n}_{1,1}$ & $\cdots \cdots$ & $\mathrm{r}_{1, j}$ & $\cdots \cdots$ & $\mathrm{r}_{1, n}$ \\
\hline$\cdots \cdots$ & $\cdots \cdots$ & $\cdots \cdots$ & $\cdots \cdots$ & $\cdots \cdots$ & $\cdots \cdots$ \\
\hline User $\mathrm{i}$ & $\mathrm{r}_{\mathrm{i}, 1}$ & $\cdots \cdots$ & $\mathrm{r}_{\mathrm{i}, \mathrm{j}}$ & $\cdots \cdots$ & $\mathrm{r}_{\mathrm{i}, \mathrm{n}}$ \\
\hline$\cdots \cdots$ & $\cdots \cdots$ & $\cdots \cdots$ & $\cdots \cdots$ & $\cdots \cdots$ & $\cdots \cdots$ \\
\hline User $\mathrm{m}$ & $\mathrm{r}_{\mathrm{m}, 1}$ & $\cdots \cdots$ & $\mathrm{r}_{\mathrm{m}, \mathrm{j}}$ & $\cdots \cdots$ & $\mathrm{r}_{\mathrm{m}, \mathrm{n}}$ \\
\hline
\end{tabular}

B. Search for nearest neighbor sets

The traditional similarity measures ${ }^{[2][6]}$ that have been used for collaborative filtering recommender systems are shown in Table II. For target user $u$, search for the nearest neighbor set which contains $k$ neighbors and denote the set by $N(u)=\left\{u_{1}, u_{2}, \ldots, u_{k-1}, u_{k}\right\}$. The value range of $\operatorname{sim}\left(u, u_{k}\right)$ is $[-1,1]$ in general where 1 is total positive correlation, 0 is no correlation, and -1 is total negative correlation. 
TABLE II. SIMILARITY MEASURES

\begin{tabular}{|c|c|}
\hline Measures & $\operatorname{sim}(u, v)$ \\
\hline Cosine ( COS ) & $\operatorname{COS}(u, v)=\frac{\sum_{i \in I_{u, v}} r_{u, i} r_{v, i}}{\sqrt{\sum_{i \in I_{u, v}} r_{u, i}^{2}} \sqrt{\sum_{i \in I_{u, v}} r_{v, i}^{2}}}$ \\
\hline $\begin{array}{l}\text { Pears on correlation } \\
\text { coefficient } \\
\text { (PCC) }\end{array}$ & $\operatorname{PCC}(u, v)=\frac{\sum_{i \in L_{u, v}}\left(r_{u, i}-r_{u}\right)\left(r_{v, i}-r_{v}\right)}{\sqrt{\sum_{i \in I_{u, v}}\left(r_{u, i}-\overline{r_{u}}\right)^{2}} \sqrt{\sum_{i \in I_{u, v}}\left(r_{v, i}-\overline{r_{v}}\right)^{2}}}$ \\
\hline $\begin{array}{l}\text { Constrained } \\
\text { Pears on correlation } \\
\text { coefficient } \\
\text { ( CPCC ) }\end{array}$ & $\operatorname{CPCC}(u, v)=\frac{\sum_{i \in I_{u, v}}\left(r_{u, i}-r_{\text {med }}\right)\left(r_{v, i}-r_{\text {med }}\right)}{\sqrt{\sum_{i \in I_{u, v}}\left(r_{u, i}-r_{\text {med }}\right)^{2}} \sqrt{\sum_{i \in I_{u, v}}\left(r_{v, i}-r_{\text {med }}\right)^{2}}}$ \\
\hline
\end{tabular}

where, $r_{u, i}$ represents the rating of item $i$ by user $u$, $I_{u, v}$ represents the common rated item set by user $u$ and $v, \bar{r}_{u}$ denotes the average rating of common rated ite $\mathrm{m}$ by user $u, r_{m e d}$ is 3 in a scale from 1 to 5 .

\section{Make recommendations}

After finding the nearest neighbor sets which was mentioned above, predict the rating of an item that user $u$ hasn't rated yet. According to result of the prediction, choose the $T o p_{n}$ items with the highest ratings and make recommendation to user himself. Prediction of a rating of an item $i$ by user $u$ can be calculated as follows:

$$
P_{u, i}=\bar{r}_{u}+\frac{\sum_{v \in N(u)} \operatorname{sim}(u, v)\left(r_{v, i}-\overline{r_{v}}\right)}{\sum_{v \in N(u)}|\operatorname{sim}(u, v)|}
$$

where, $\bar{r}_{u}$ represents the average rating of rated item by user $u, N(u)$ represents the nearest neighbor set of user $u$, $\operatorname{sim}(u, v)$ denotes the similarity between user $u$ and $v$, $r_{v, i}$ denotes the rating of item $i$ by user $v$.

\section{COLLABORATIVE FILTERING ALGORITHM COMBINED WITH USER HABITS FOR RATING}

Considering that everyone has his own habit, people with similar habits are generally similar in a certain degree. Therefore a novel algorithm combined with user habits for rating is put forward which takes both traditional measure and user habits into account. In a rating system, each user has his unique rating habit. Some of the users are accustomed to giving high ratings, and some users have a much more centralized distribution of ratings than others. For example, the rating vectors of $u_{1}, u_{2}$ and $u_{3}$ are $(1,0,2,0,1,0,2,0) \quad, \quad(0,1,0,2,0,1,0,2) \quad$ and $(0,5,0,4,0,4,0,3)$. In order to reveal the hidden relationship between users, Bhattacharyya coefficient and entropy are introduced.

\section{A. Bhattacharyya coefficient}

Bhattacharyya coefficient is an approximate measurement of the amount of overlap between two statistical samples or populations. It is widely used in signal processing, pattern recognition and other professional fields ${ }^{[7]}$. For discrete domain $X$, it is defined as:

$$
B C(p, q)=\sum_{x \in X} \sqrt{p(x) q(x)}
$$

where $p(x)$ and $q(x)$ represent the discrete probability distributions of $p$ and $q$ relatively. Supposing that $p_{u}$ and $p_{v}$ are the estimated discrete densities of user $u$ and $v$. BC similarity between user $u$ and $v$ is computed as:

$$
B C(u, v)=\sum_{i=1}^{n} \sqrt{p_{u, i} p_{v, i}}
$$

where, $n$ represents the number of partitions, $p_{u, i}$ represents the ratio of the number of items with rating value $i$ by user $u$ to the total number of items rated by user $u, \sum_{i=1}^{n} p_{u, i}=\sum_{i=1}^{n} p_{v, i}=1$.

This can be illustrated with an example. Let $u_{1}=(1,0,2,0,1,0,2,0)$ and $u_{2}=(0,1,0,2,0,1,0,2)$ be the rating vectors of user $u_{1}$ and $u_{2}$. The ratings lie in $\{1,2\}$, hence the number of partitions is 2. BC coefficient between user $u_{1}$ and $u_{2}$ :

$$
\begin{aligned}
B C\left(u_{1}, u_{2}\right) & =\sum_{i=1}^{2} \sqrt{p_{u_{1}, i} p_{u_{2}, i}} \\
& =\sqrt{\frac{2}{4} \times \frac{2}{4}}+\sqrt{\frac{2}{4} \times \frac{2}{4}}=1
\end{aligned}
$$

It can be noted that there is no common rated items between $u_{1}$ and $u_{2}$. Existing conventional measures could not calculate user similarity in this scenario. It also can be noted that $u_{1}$ and $u_{2}$ both have preferences for giving low ratings. In addition to that, two users have an identical rating distribution which can be inferred that $u_{1}$ and $u_{2}$ are similar in rating habits. Let $u_{3}=(0,5,0,4,0,5,0,3)$ be the rating vector of user $u_{3}$. A problem occurs where $\mathrm{BC}$ coefficient between $u_{1}$ and $u_{3}$ equals 0 because there is no overlap at all in every partition. From the perspective of dispersion degree of ratings, $u_{1}$ and $u_{3}$ still have a certain similarity as their ratings are relatively centralized in distribution. Consequently, entropy is introduced to measure the rating dispersion degree to settle this problem.

\section{B. Entropy of information(Shannon entropy)}

The father of information theory Shannon introduced entropy of information in his 1948 paper which was borrowed from the concept of thermodynamics. As is known that in a thermodynamic system, entropy is a measure of disorder or chaos in the universe. Shannon defined entropy of information as the uncertainty of source by analogy ${ }^{[8-10]}$. Assuming that the source has $n$ kinds of possible values, the corresponding probability for each value is $p_{1}, p_{2}, \cdots, p_{n}$, entropy of information is defined as:

$$
H(U)=-\sum_{i=1}^{n} p_{i} \log _{2} p_{i}
$$


TABLE III. SIMILARITY MEASURES

\begin{tabular}{|c|c|c|c|c|c|c|}
\hline $\begin{array}{c}\text { User } \\
\text { No. }\end{array}$ & $\begin{array}{c}\text { No. of } \\
1\end{array}$ & $\begin{array}{c}\text { No. of } \\
2\end{array}$ & $\begin{array}{c}\text { No. of } \\
3\end{array}$ & $\begin{array}{c}\text { No. of } \\
4\end{array}$ & $\begin{array}{c}\text { No. of } \\
5\end{array}$ & Entropy \\
\hline 60 & 0 & 0 & 16 & 71 & 32 & 1.34 \\
\hline 186 & 4 & 8 & 13 & 14 & 9 & 2.21 \\
\hline 631 & 0 & 7 & 4 & 9 & 0 & 1.51 \\
\hline
\end{tabular}

As Table III. shows that, a certain user with higher Shannon entropy has relatively decentralized rating distribution. Like wise, a user with centralized distribution of rating has comparatively lower entropy. Entropy similarity between $u_{1}$ and $u_{2}$ is computed as:

$$
\operatorname{En}\left(u_{1}, u_{2}\right)=\exp \left(-\left|H\left(u_{1}\right)-H\left(u_{2}\right)\right|\right)
$$

It can be seen from the formula above that users are more similar when they have smaller difference in Shannon entropy.

\section{Collaborative Filtering Algorithm Combined with User Habits for Rating}

A new algorith $m$ combined with user habits for rating is proposed for the sake of mining the information contained in user ratings. Bhattacharyya coefficient and entropy are separately presented in sections above, the similarity between $u_{1}$ and $u_{2}$ based on rating habits are defined as:

$$
\operatorname{Habit}\left(u_{1}, u_{2}\right)=\left\{\begin{array}{cl}
E n\left(u_{1}, u_{2}\right) & B C\left(u_{1}, u_{2}\right)=0 \\
\sqrt{\operatorname{En}\left(u_{1}, u_{2}\right) \times B C\left(u_{1}, u_{2}\right)} & B C\left(u_{1}, u_{2}\right) \neq 0
\end{array}\right.
$$

Both traditional measures and user habits for rating are considered in this paper. Therefore, with the new measure, the similarity between two users $u_{1}$ and $u_{2}$ is calculated as:

$$
\operatorname{BCE}\left(u_{1}, u_{2}\right)=\operatorname{Habit}\left(u_{1}, u_{2}\right)+\operatorname{PCC}\left(u_{1}, u_{2}\right)
$$

It can be noted that user similarity is determined by Pearson correlation coefficient and user habits for rating. If there exists two users who have close PCC with a certain user $u$, the one who has larger Habit with user $u$ is more similar than the other one and vice versa.

From the formula above, researchers can know that the value range of $\operatorname{Habit}\left(u_{1}, u_{2}\right)$ is 0 to 1 and the value range of $\operatorname{PCC}\left(u_{1}, u_{2}\right)$ is -1 to 1 . Hence, the value range of $\operatorname{BCE}\left(u_{1}, u_{2}\right)$ is -1 to 2 . However, the value range of most traditional measures is -1 to 1 . Modify the BCE equation by linear mapping to adjust its value between -1 and 1 , the equation is modified as follows:

$$
N B C E[i]=-1+\frac{2 \times(\operatorname{Max}(B C E)-B C E[i])}{\operatorname{Max}(B C E)-\operatorname{Min}(B C E)}
$$

where $\operatorname{Max}(B C E)$ and $\operatorname{Min}(B C E)$ represents the maximum and minimum value in $B C E$. After modification, the value of 1 is total positive correlation, 0 is no correlation, and -1 is total negative correlation. This paper considers $N B C E$ to be the new method of measuring usersimilarity.

\section{EXPERIMENTS}

The simulation environment includes: the $\mathrm{PC}$ with Windows 7, Intel ${ }^{\mathrm{TM}}$ Core $^{\mathrm{TM}} 2$ Duo CPU E7400 $2.80 \mathrm{GHz}$ CPU, 4.00GB RAM. The new algorithm is coded by Python.

\section{A. Dataset and evaluation metrics}

We use the MovieLens ${ }^{[1]}$ dataset which was created by GroupLens Research in our experiments. GroupLens provides three datasets of different size, in this experiment, researchers use the ML-100k as the dataset. It includes 943 users and 1682 movies with 100000 ratings within 7 months. Each person has rated at least 20 movies and the movie includes 19 types. The dataset was randomly split into training and test data respectively with a ratio of $80 \% / 20 \%$. The sparsity level of dataset is 1$100000 /(943 * 1682)=93.7 \%$.

We employed the mean absolute error (MAE) and root mean square error (RMSE) metrics to measure the predit quality of proposed approach and compare the $N B C E$ algorithm with traditional ones. MAE and RMSE are accuracy metrics which measure the difference between the predicted ratings and actual ratings on test users. A lower MAE or RMSE value corresponds to more accurate predictions. MAE and RMSE are defined as follows:

$$
\begin{array}{r}
M A E=\frac{\sum_{(u, i) \in I_{T}}\left|p_{u, i}-r_{u, i}\right|}{\left|I_{T}\right|} \\
R M S E=\sqrt{\frac{1}{\left|I_{T}\right|} \sum_{(u, i) \in I_{T}}\left(p_{u, i}-r_{u, i}\right)^{2}}
\end{array}
$$

where, $\left|I_{T}\right|$ represents the number of ratings in test data, $p_{u, i}$ denotes the predicted rating of item $i$ by user $u, r_{u, i}$ denotes the actual rating of item $i$ by user $u$.

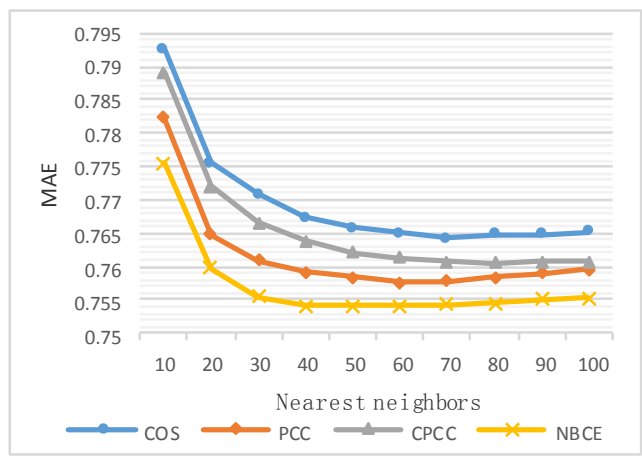

Figure 1. MAE value comparison bet ween NBCE and other traditional methods

\section{B. Experiment results and analysis}

In order to verify the effectiveness of the new method, researchers compare $N B C E$ with traditional $\mathrm{CF}$ algorithms. 


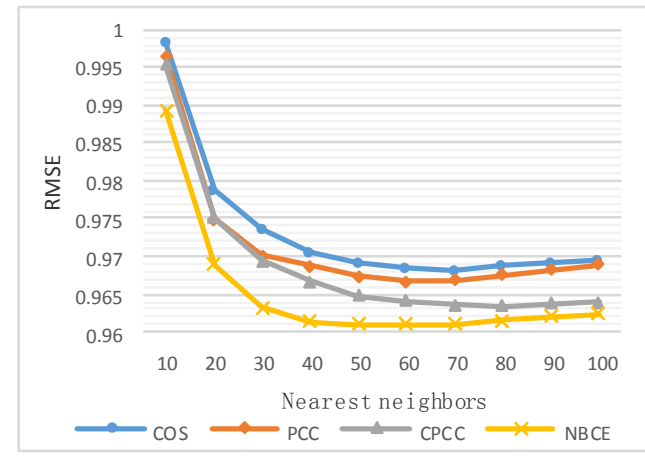

Figure 2. RMSE value comparison bet ween NBCE and other traditional methods

Fig. 1 and Fig. 2 respectively show the MAE and RMSE value curves along with the number of nearest neighbors. The curves of $N B C E$, the same as the other conventional approaches, decreases rapidly with the increasing number of nearest neighbors and flattens out slowly. MAE and RMSE value are smaller than that of the traditional methods as $N B C E$ additionally takes user habits for rating into account which has better performance in recommendation.

\section{Complexityanalysis}

As Bhattacharyya coefficient and entropy are applied to $\mathrm{CF}$ algorith $\mathrm{m}$ combined with user habits for rating, the complexity of the user similarity calculation improves in some degree. Supposing there exists $m$ users and $n$ items in a recommender system, user-item rating matrix is needed to be built in the traditional measures as well as the new approach. The time and space complexity are both $O(m n)$. In the stage of searching for nearest neighbors, the traditional methods only need to calculate the similarity between users. The time and space complexity are both $O\left(\mathrm{~m}^{2}\right)$. Besides that, the new measure requires computing the Bhattacharyya coefficient and entropy and storing the data although they can be collected during the process of building the matrix beforehand. Thus the time and space complexity still remains $O\left(\mathrm{~m}^{2}\right)$. On the whole, the complexity of new algorithm increases in both time and space, but it has little effect on the recommendation system.

\section{CONCLUSIONS}

For the purpose of exploring the "hidden" relationship between users, a new collaborative filtering algorithm combined with user habits for rating is put forward which not only considers the traditional measure but utilizes the Bhattacharyya coefficient and Shannon entropy to compute the user rating habits. The new approach outperforms the traditional method with the combined action of the two factors mentioned above. The effect of the proposed algorithm in different evaluation criteria (e.g., Precision, Recall, etc) and other data sets needs to be further studied and verified. In addition, user's interest tends to constantly change according to the factors of time and space which may increase the complexity of calculating user similarities, and how to improve the systemscalability needs to be studied next.

\section{ACKNOWLEDGMENT}

The authors would also like to thank all the participants who gave cooperation in the experiments. This work was partially supported by National High-tech R\&D Program of China (NO.2013AA01A211).

\section{REFERENCES}

[1] Adomavicius G, Tuzhilin A. Toward the next generation of recommender systems: A survey of the state-of-the-art and possible extensions[J]. Knowledge and Data Engineering, IEEE Transactions on, 2005, 17(6): 734-749.

[2] Sarwar B, Karypis G, Konstan J, et al. Item-based collaborative filtering recommendation algorithms[C]/Proceedings of the 10 th international conference on World Wide Web. ACM, 2001: 285295.

[3] Koren Y, Bell R, Volinsky C. Matrix factorization techniques for recommender systems[J]. Computer, 2009(8): 30-37.

[4] Ahn H J. A new similarity measure for collaborative filtering to alleviate the new user cold-starting problem $[\mathrm{J}]$. Information Sciences, 2008, 178(1): 37-51.

[5] Amatriain X, Lathia N, Pujol J M, et al. The wisdom of the few: a collaborative filtering approach based on expert opinions from the web[C]//Proceedings of the 32nd international ACM SIGIR conference on Research and development in information retrieval. ACM, 2009:532-539.

[6] Deng Ai-Lin, Zhu Yang-Yong, Shi Bai-Le. A Collaborative Filtering Recommendation Algorithm Based on Item Rating Prediction[J]. Journal of Soft ware, 2003, 14(9): 1621-1628.

[7] Khalid M S, Ilyas M U, Sarfaraz M S, et al. Bhattacharyya coefficient in correlation of gray-scale objects[J]. Journal of Multimedia, 2006, 1(1): 56-61.

[8] Chun-Hui Piao, Jing Zhao, Li-Juan Zheng. Research on entropybased collaborative filtering algorithm and personalized recommendation in e-commerce [J]. Service Oriented Computing and Applications, 2013,3(2):147-157.

[9] Kaleli C. An entropy-based neighbor selection approach for collaborative filtering[J]. Knowledge-Based Systems, 2014, 56: 273-280.

[10] Mehta H, Dixit V S, Bedi P. Weighted difference entropy based similarity measure at two levels in a recommendation framework[C]//Advances in Computing, Communications and Informatics (ICACCI), 2013 International Conference on. IEEE, 2013: 2076-2083

[11] MovieLens[EB/OL]. http://grouplens.org/datasets/movielens/. [2015-4-25]. 